\title{
La segmentación territorial de la educación general básica y el pueblo indigena kichwa kisapincha de Ambato, Tungurahua en Ecuador
}

Territorial Division of Basic General Education and The Kichwa Kisapincha Natives from Ambato, Tungurahua in Equator

A segmentação territorial da educação básica geral e os povos indígenas Kichwa Kisapincha de Ambato, Tungurahua no Equador

Judith Pinos-Montenegro* iD orcid.org/0000-0003-3478-2082

Para citar este artículo: Pinos-Montenegro, J. (2020). La segmentación territorial de la educación general básica y el pueblo indígena kichwa kisapincha de Ambato, Tungurahua en Ecuador. Revista Colombiana de Educación, /(80), 313-334. https://doi.org/10.17227/rce.num80-10799 


\section{Resumen}

El presente artículo es el resultado final de una investigación de campo con enfoque participativo. Tiene por objetivo identificar y describir las ideas sobre la segmentación de los servicios educativos que manifiestan agentes indígenas. Teóricamente, este trabajo usa los pensamientos de Weber, Bourdieu, Ringer y Archer, que se enfocaron en la capacidad de agencia de quienes confluyen en un contexto social. Metodológicamente es una investigación cualitativa de tipo exploratorio y un estudio de caso. La indagación se realizó durante los años 2017 a 2019. Los hallazgos principales muestran que en el 2017 la segmentación territorial de los servicios escolares fue percibida como una arbitrariedad del Estado, que incumplió con el mandato constitucional de consulta previa y afectó el acceso a la educación. Sin embargo, hoy la política pública es parcialmente aceptada y lo que se cuestiona es la poca pertinencia cultural de los servicios educativos.

\section{Palabras clave}

educación básica; política pública; población indígena acceso a la educación; pertinencia cultural

\section{Keywords}

basic educación; policy public; indigenous populations; educational access; cultural relevance

\section{Abstract}

The article is the result of a field research with a participatory approach. This aims to identify and describe the ideas that native's agents manifest about the segmentation of educational services. Theoretically, this work uses the thoughts of Weber, Bourdieu, Ringer and Archer, who focused on the agency of those who come together in a social context. Methodologically, it is a qualitative, exploratory case study. The research was conducted from 2017 to 2019. The main findings of this work show that, in 2017, the territorial segmentation of school services was perceived as an arbitrary decision of the State, which did not meet the constitutional mandate of prior consultation, and affected access to education. Even though today public policy is partly accepted, people question the lack of cultural relevance of educational services.

\section{Resumo}

Este artigo é o resultado de uma pesquisa de campo com abordagem participativa. Seu objetivo é identificar e descrever as ideias sobre a segmentação dos serviços educacionais que os agentes indígenas manifestam. Teoricamente, este trabalho usa o pensamento de Weber, Bourdieu, Ringer e Archer, que enfocaram a capacidade de agência daqueles que se reúnem em um contexto social. Metodologicamente é uma pesquisa exploratória qualitativa e um estudo de caso. A investigação foi realizada durante os anos de 2017 a 2019. Os principais achados mostram que em 2017 a segmentação territorial dos serviços escolares foi percebida como arbitrária pelo Estado, que não cumpriu o mandato constitucional de consulta prévia e afetou o acesso à educação. Porém, hoje as políticas públicas são parcialmente aceitas e o que se questiona é a falta de relevância cultural dos serviços educacionais.

\section{Palavras-chave}

educação básica; políticas públicas; população indígena; acesso à educação; relevância cultural 


\section{Introducción}

Esta investigación se preguntó cómo recibieron los indígenas kichwas el sistema de segmentación territorial educativa para los servicios estatales ubicados en la parroquia Kisapincha, del cantón Ambato, de la provincia Tungurahua (Ecuador), política implementada desde el 2011. Esta pregunta central se deriva a su vez en dos interrogantes: 1) ¿Qué cambios perciben los pueblos indígenas respecto al acceso a la educación, tras el proceso de segmentación territorial? 2) ¿Qué aspectos en torno a la segmentación educativa reclaman los pueblos indígenas?

Antes del 2012, los servicios educativos en Ecuador se organizaban territorialmente a nivel macro en direcciones provinciales y a nivel micro, en jardines, escuelas y colegios. El cuerpo docente laboraba cuatro horas diarias en forma presencial en su lugar de trabajo. En el 2011, la Asamblea Nacional sancionó la Ley Orgánica de Educación Intercultural (LOEI); al año siguiente se expidió el Registro Oficial 290, que dividió el territorio a nivel macro en zonas, distritos y circuitos escolares y a nivel micro en unidades educativas (UE). La transformación se implementó paulatinamente con la agrupación de docentes y estudiantes (de 5 a 18 años de edad) en las unidades. Se dispuso al personal docente que labore en jornadas de ocho horas diarias de forma presencial en las UE (Pinos Montenegro, 2017). Este escrito se centra en la mirada de la población indígena usuaria del servicio (padres, madres de familias y líderes indígenas).

El país reconoce oficialmente la existencia de 18 pueblos y 14 nacionalidades indígenas (Secretaría Nacional de Planificación del Desarrollo- Senplades, 2017), de las cuales la kichwa es la más numerosa y a esta pertenece el pueblo kisapincha ${ }^{1}$ (Confederación de Nacionalidades Indígenas del Ecuador-Conaie, 2018). Según el último censo, en Ecuador hay 14483499 habitantes, pero solo el 6,8 \% se autoidentifican como indígenas (Instituto Nacional de Estadísticas y Censos -INEC, 2010). La provincia de Tungurahua tiene 504583 habitantes y el 12,4\% de ellos se reconoce indígena, es decir, casi el doble en comparación con el dato nacional. Tungurahua está en la sierra andina y se divide en nueve cantones; en el cantón Ambato está la parroquia Kisapincha, a 3000 m s. n. m. Esta parroquia posee un clima frío; es muy antigua, existe desde el año 1570 (GAD Kisapincha, 2015); tiene 13001 habitantes (6235 varones y 6766 mujeres), de los cuales el 70,86 \% se identifica como indígena kichwa kisapincha,

1 Kisapincha es una palabra que tiene variantes en sus usos y registros. Es el nombre de la parroquia del cantón Ambato, lugar que estudiamos, la parroquia Kisapincha. Es el nombre del pueblo kichwa kisapincha, y es el nombre de una de las UE, que lo registra como Quizapincha. 
el 1,28 \% como blancos y el resto como mestizos (INEC, 2010). Esa mayoría étnica tiene un contexto cultural y lingüístico específico, marcado por el kichwa como lengua materna y el español como lengua dominante.

Según la Conaie,

[los] Kichwas de la sierra ecuatoriana están vinculados por el idioma kichwa, por un territorio que se les fue desapropiado en tiempos de la colonia española, por una misma cosmovisión en relación al territorio y al universo, por los vínculos comerciales que existían antes de la venida de los españoles. (2018, p. 3)

Es decir, los pueblos kichwas no solo comparten el territorio, sino que son portadores de una cultura común; lo kichwa alude a la etnia, a la cultura y al idioma. Para Haboud (1998) el kichwa tiene raíces en el quechua del incario, y fue modificado a través de los años. En la Constitución (2008), el kichwa se registra como un idioma oficial y de intercambio cultural, que debe ser promovido y fortalecido.

Los pueblos indígenas de la provincia se agrupan en la Unidad de Movimientos Indígenas y Campesinos de Tungurahua (Unmict). En el 2017 se contactó a esta organización para solicitar su participación y asentimiento en esta investigación. Durante este tiempo (desde el año 2017 hasta el 2019) se discutió detalladamente la perspectiva de trabajo y se recogió información; gracias a la mediación de Unmict se contactó a líderes de comunidades y padres/madres de familia que usan las UE.

La población de la parroquia Kisapincha se distribuye en dos grandes grupos. El primero, en la parte central del territorio parroquial, donde está cerca del $25 \%$ de los habitantes. Allí predomina el español y sus habitantes se autoidentifican como mestizos; la conforman doce barrios (la Plaza, Cashauco, la Alborada, el Centro, el Mirador, el Panecillo, Turuloma, Pasañán, la Calera, San Pedro, Santa Elena y el Calvario). El segundo grupo está en la zona más montañosa; sus integrantes se reconocen como indígenas y habitan en 18 comunidades (Calhuasig Chico, Calhuasig Grande, Illagua Chico, Illagua Chupiloma, Illagua Grande, Nueva Tondolique, el Galpón, Putugleo, Ambayata, Pukará Chico, Pukará Grande, Cachilvana Grande, Cachilvana Chico, Puganza, Santa Rosapamba, Condezán, Quindialó y Santa Cruz de Chumilca). En el centro de la parroquia se usa el idioma español y en las 18 comunidades predomina el kichwa.

Si bien la LOEl se sancionó en el año 2011 y determinó la segmentación territorial, el cambio estructural fue paulatino y recién en el 2014 la parroquia Kisapincha pasó de tener quince escuelas a contar con cuatro (ue Quizapincha, ue del Milenio Pueblo Kisapincha, ue Casahuala y UE Ciudad de Azoguez). En esta investigación nos preguntamos cómo fue recibida dicha política pública. 
En cuanto a las condiciones educativas y sociales que rodean a estas poblaciones, según Mato (2015), la población indígena afronta problemáticas particulares como la "discriminación racial, padecen un sinfín de consecuencias prácticas asociadas a diferencias lingüísticas y generalmente viven en condiciones económicas desventajosas" (p. 12). En Ecuador, diversos informes del Instituto Nacional de Evaluación (Ineval) evidencian que las poblaciones indígenas obtienen menores resultados de aprendizaje en comparación con el resto de la población (Ineval, 2016; 2018). En palabras de Ponce Jarrín y Acosta (2010), a pesar de los esfuerzos estatales, la pobreza sigue afectando a los pueblos indígenas y las políticas sociales implementadas a partir del 2007 carecerían de la suficiente fuerza como para superar la desigualdad y exclusión que afecta a las poblaciones indígenas. Algunos estudios (Di Caudo et al., 2016) apuntan a que dicha situación se produce debido a la baja la calidad y a la poca pertinencia cultural de los servicios públicos; las investigaciones mencionadas analizaron la década comprendida entre 2007 y 2017.

Según la versión gubernamental, a partir del 2007 se otorgó mayor presupuesto para atender al sector de la educación (Ineval, 2016, Senplades, 2017). Con más recursos, entre 2007 y 2017 se implementó una reforma sustancial a todo el sistema educativo, en los niveles, la rectoría estatal, los currículos, los instrumentos de medición de calidad, la evaluación docente y la producción de materiales educativos (Isch, 2011; Luna Tamayo, 2009). Pero, también se produjeron continuidades, como que el sistema educativo se divide en dos grandes subsistemas: uno para atender a población no indígena y otro para la indígena (este último es el Sistema de Educación Intercultural Bilingüe-SEIB). En tal sentido, el marco legal es igual al anterior (Ley de Educación 127 de 1989 y la Constitución de 1998) pues mantiene la división física y simbólica entre lo indígena y lo no indígena.

La actual Constitución reconoce los derechos de pueblos y nacionalidades indígenas, entre estos los culturales, organizativos y ciudadanos. Establece la obligación, del Estado y de toda instancia, de consultarles sobre cualquier "potencial afectación ambiental o cultural" (artículo 57 de la Constitución, 2008). Sin embargo, en el mismo cuerpo legal se dictamina que la educación y su administración son potestad exclusiva del Estado central.

Tras el nuevo marco legal, la educación obligatoria se incrementó de seis a diez años, es decir, se pasó a exigir a las familias que la población de 5 a 15 años de edad reciba educación, y se la llamó Educación General Básica; aspecto que es positivo porque mejora el promedio de escolaridad de la población ecuatoriana. Para ampliar la obligatoriedad y brindar educación completa se eliminaron jardines, escuelas y colegios y se fusionó el servicio bajo la figura de las UE, que atienden a estudiantes desde los 5 años hasta los 18 años (en los mismos edificios escolares). 
La estrategia estatal fue concentrar estudiantes y docentes, y cerrar escuelas con un docente para todos los niveles educativos y/o varios estudiantes de diversos grados (escuelas unidocentes y multigrado). Cabe aclarar que el personal docente de las escuelas cerradas no fue despedido, sino trasladado. Esta medida, desde el punto de vista lógico puede parecer acertada y hasta deseada, porque brinda educación completa y optimiza recursos. No obstante, recibió el cuestionamiento de los pueblos indígenas y de instancias como la Cepal, que en el estudio de León (2017) da cuenta del malestar de los sectores rurales:

Varios entrevistados para este informe mencionan información cualitativa que indica que el modelo de cierre de escuelas rurales, planificado centralmente, no consideró adecuadamente la realidad de las distancias entre algunas de las escuelas a cerrarse y las nuevas, ni la existencia de vías apropiadas de comunicación y transporte, lo que genera atrasos y problemas en la asistencia escolar. (p. 68)

El surgimiento de la política de segmentación territorial de los servicios educativos, expresada en la conformación de las UE, donde se produjo la concentración de estudiantes y docentes, Ilevó a la implementación de medidas específicas, las cuales desde el punto de vista legal se registraron así:

Art. 26.- La educación es un derecho de las personas a lo largo de su vida y un deber ineludible e inexcusable del Estado. Constituye un área prioritaria de la política pública y de la inversión estatal, garantía de la igualdad e inclusión social y condición indispensable para el buen vivir. Las personas, las familias y la sociedad tienen el derecho y la responsabilidad de participar en el proceso educativo. (Constitución, 2008)

Art. 6.- Obligaciones.- La principal obligación del Estado es el cumplimiento pleno, permanente y progresivo de los derechos y garantías constitucionales en materia educativa, y de los principios y fines establecidos en esta Ley.

El Estado tiene las siguientes obligaciones adicionales:

- Garantizar, bajo los principios de equidad, igualdad, no discriminación y libertad, que todas las personas tengan acceso a la educación pública de calidad y cercanía. (LOEI, 2011. Énfasis añadido).

Art. 2. Fortalézcase el Sistema Informático de Asignación de Cupos del Ministerio de Educación en Coordinación con el Sistema de la Dirección General del Registro Civil, Identificación y Cedulación (Digercic). Para tal efecto en el proceso de inscripción y posterior asignación de cupos, para la oferta educativa, se requerirá que el aspirante cuente con el número de cédula generado por la Digercic (Acuerdo del Ministerio de Educación, 353-13, expedido el 16 de septiembre de 2013) 
En lo que antecede, se evidencia un proceso paulatino de ordenamiento, automatización, derivación y distribución de estudiantes a escala nacional. Así, mientras que en el 2008 la Constitución ecuatoriana sostuvo que la educación es un derecho y el Estado es su garante, en el 2011 expidió la Ley Orgánica de Educación Intercultural, que en su artículo 6, literal b, señala la "cercanía" como un principio que condiciona el derecho a la educación pública, se refiere a la cercanía de la vivienda y el servicio educativo. En el 2013, empezó la asignación automática de cupos escolares, como lo expresa el Acuerdo 353-13. En la actualidad, el sistema es totalmente virtual y tiene su propio portal (https://juntos.educacion.gob.ec/index.php/consulta-sierra/ asignacion). El proceso instalado mantiene algunas dificultades, según la prensa nacional ("Agrupación de hermanos en planteles fiscales depende de disponibilidad de cupos, dice Ministerio de Educación", 2018; "Problemas con asignación de cupos en escuelas persisten", 2019) y como lo había documentado la Cepal en el informe ya mencionado.

Desde la implementación de dichas disposiciones legales, entre 2008 y 2018, las organizaciones indígenas, agrupadas en la Conaie, pidieron la restitución de escuelas localizadas en zonas rurales y creadas con la participación comunitaria (Conaie, 2018). El reordenamiento de servicios educativos públicos, basado en las perspectivas de la eficiencia y la eficacia administrativa inicialmente causó incomodidad en los pueblos indígenas que reclamaban la permanencia de servicios educativos creados en comunidades rurales. Resultó evidente una tensión respecto al hecho educativo, en particular a la política de segmentación. Mientras para el Estado la sectorización fue un proceso administrativo que ahorró tiempo y recursos y permitió una mejor planificación del sistema educativo, para los pueblos indígenas fue una arbitrariedad.

\section{Decisiones conceptuales y metodológicas}

En esta investigación se emplean los conceptos segmentación educativa, acceso físico, acceso cultural y agentes. Para abordar el concepto de segmentación educativa, en este trabajo se sigue el pensamiento de Weber, Bourdieu, Ringer y Archer, en ese orden. Así, resulta útil el planteamiento de Ringer (1990), quien combinó los aportes de Bourdieu (1970) y de Weber (1964) para analizar la segmentación educativa de los casos europeos y de Estados Unidos de América, de fines del siglo xix. De Bourdieu se rescata la diferencia entre capital cultural y capital económico (Bourdieu y Passeron, 1970); de Weber, los conceptos de clases y status, resaltando que el status permite pensar la valoración y el prestigio. Así, tras una conjugación de ambas ideas, Fritz Ringer señala que los servicios escolares están bañados de diferente valor simbólico y prestigio, al igual que los aspirantes al uso de esos servicios, como portadores de una gama 
de capitales económicos y culturales. Por lo tanto, el contexto es como un tapiz donde hay instituciones educativas más deseables que otras y aspirantes con diferente capital social (cultural y económico).

Operativamente, Ringer propone centrar la atención en dos aspectos: el porcentaje de acceso y la probabilidad de acceso. En su lectura, la segmentación de los servicios educativos alude a la diversidad de instituciones educativas que pueden ser asociadas en segmentos pero que son diferentes entre sí, ya sea por la población de estudiantes, por su origen de clase o por el tipo de oferta curricular. Por su parte, Margaret Archer (2006) señala que todo hecho social debe ser leído a la luz de sus antecedentes y que ni la estructura es totalmente determinante en el destino de las personas y de los grupos sociales, ni estos son totalmente libres de actuar sin referencia a la estructura social. Para el caso en estudio se acogió esta última perspectiva.

En esta investigación, se asume que el acceso es "un proceso mediante el cual se puede lograr que una necesidad de atención se satisfaga completamente" (Fuentes Reverón, 2017, p. 273). Para el caso que estudiamos, los pueblos indígenas demandan, por un lado, acceso a educación, y por otro, una educación con pertinencia cultural que fortalezca su idioma nativo. Como ya se mencionó, para Ringer (1990) hay que distinguir el acceso de la probabilidad de acceso. En el contexto actual ecuatoriano resulta útil distinguir algunos aspectos:

» La educación general básica es obligatoria.

»El Estado central es el garante del acceso a la educación general básica, pero este está condicionada a la cercanía del servicio educativo y la vivienda del aspirante a satisfacer esa necesidad.

» Estado central es el controlador y el habilitador del acceso a la educación.

»El Estado asigna el lugar para estudiar, a la persona que desea (utilizar) un servicio educativo público. Mientras, que quien puede pagar una educación privada, puede moverse libremente por el territorio.

De diversos estudios sobre segmentación educativa (Krüger, 2012; Massey, 2008; Verhoeven, 2013), se puede deducir que el Estado tiene la posibilidad de facilitaro dificultar el acceso con varias medidas, entre otras la segmentación territorial (Dubet, 2011). Esta implica el cierre, la fusión o la apertura de servicios educativos.

En el caso de esta investigación el acceso se refiere al acceso a recursos de aprendizaje, particularmente, a recursos en lengua materna o en lengua de las nacionalidades indígenas. De ahí que, si bien alguien puede tener acceso físico a un servicio educativo, este puede ser cercano o lejano culturalmente hablando. 
El aspecto físico se refiere a la distancia percibida entre el lugar del servicio educativo y el sitio del que parte (sale) quien desea cubrir esa necesidad. El cultural es la cercanía o no al marco lingüístico, de costumbres y saberes, que para el caso ecuatoriano está indicado en los derechos culturales de los pueblos y nacionalidades indígenas y reivindicados también por diferentes tratados internacionales (la Convención Internacional para la Eliminación de Todas las Formas de Discriminación Racial, 1965; la Convención Internacional sobre Derechos Económicos, Sociales y Culturales, 1966; el Convenio 169 de la Organización Internacional del Trabajo, 1968). Así, se deduce que puede haber acceso total y acceso parcial a un servicio educativo.

Por lo tanto, aunque la creación de una política pública educativa, como la de segmentación territorial del servicio educativo, está dirigida por igual a los usuarios de los servicios públicos, se requiere un elemento adicional de análisis: la diferenciación entre agentes; es decir, no todos viven en iguales condiciones. Para Archer (2017) pueden identificarse tres aspectos en el vínculo entre agentes, recursos y capacidad de interactuar con la instancia de decisión:

» Los agentes con poco acceso a recursos están en la posición negociadora más débil.

» Los agentes con acceso diferente a recursos están en una posición negociadora más fuerte.

» Los agentes con más acceso a los recursos tienen la mejor posición negociadora.

Al aplicar las ideas de Archer (2017) a esta investigación, se infiere que quienes no tienen recursos económicos para optar por un tipo de educación que no sea la gratuita (pública) están en menos condiciones de negociar, porque se ubicarían en una posición más débil con relación a la decisión del Estado. Sin embargo, las comunidades indígenas poseen una ventaja importante: están organizadas a niveles comunitarios y a nivel nacional. Durante diferentes periodos históricos, los gobiernos de turno han tenido que negociar con ellas; por ejemplo, en los levantamientos indígenas de los años 1990, 2009 y 2019. Además, es preciso considerar que en el contexto ecuatoriano debe ejecutarse la "consulta previa", como lo establece el artículo 57, parágrafo 7 de la Constitución del 2008, ya que un cambio en la estructura educativa afecta directamente los derechos culturales de los pueblos indígenas.

En consonancia con lo expuesto y con el objetivo de esta investigación centrado en comprender la perspectiva de los agentes, se optó metodológicamente por una perspectiva cualitativa. Es un estudio de caso, que como lo define Stake: "[...] es el estudio de la particularidad y de la complejidad de un caso singular, para llegar a comprender su actividad en 
circunstancias importantes" (2005, p. 8). El tipo de caso es considerado por Hernández Sampieri et al. (2012) como un camino cualitativo, que permite descubrir y entender cómo los agentes perciben los acontecimientos. Este estudio de caso es de aproximación metodológica, que, en palabras de Vasilachis de Gialdino (2013) "pueden ser definidos como estrategias de investigación empírica [...] se diferencian del caso en sí mismo o del estudio del caso simplemente" (p. 222). Así, se deduce que el estudio de caso como aproximación metodológica permite la comprensión del contexto y de las interpretaciones de los agentes.

Es una indagación exploratoria, pues la política de segmentación territorial educativa es relativamente nueva en Ecuador y, en particular, los análisis sobre el tema son mínimos puesto que no existen indagaciones previas en la población de la parroquia Kisapincha. A su vez, este trabajo académico recoge percepciones de un grupo humano particular, cuyos miembros se autoidentifican como indígenas kichwas. El proceso tuvo un enfoque participativo y siguió estos pasos:

» Se consultó a la Unmict sobre la posibilidad de llevar a cabo esta investigación. Ellos, a su vez, consultaron con las instancias comunitarias, que deliberaron y, en mayo del 2017 dieron su consentimiento y asentimiento por escrito. Como resultado, se tuvo acceso a la zona indígena y se tuvo contacto con líderes comunitarios de la parroquia Kisapincha.

» El trabajo de campo se realizó durante el 2017 y, posteriormente, en el 2019.

» En el 2017 se acordó un calendario de visitas a la parroquia. Se desplegó un trabajo paulatino en las zonas campesinas de las comunidades de Kisapincha.

» Se grabaron las entrevistas y se sistematizaron usando la perspectiva de Coffey y Atkinson (1996) de identificación de códigos.

» Al finalizar el 2017, se decidió extender la investigación, pues se aproximaba un cambio en el contexto nacional y particularmente de gobierno.

» Durante el 2018 se observó el comportamiento de los grupos sociales y sus demandas sobre la educación prevalecieron, aunque poco a poco presentaron menor intensidad.

» En el 2019, la relación social entre las organizaciones indígenas y el actual Gobierno nacional (presidido por Lenin Moreno) se tensó. Por esa razón, se decidió volver a entrevistar a los líderes comunitarios y al Gobierno parroquial de Kisapincha.

» Los resultados de ambos procesos de entrevistas se consolidaron en cuatro unidades de análisis: cierre de escuelas e identidad, 
segmentación del territorio y acceso a educación, derechos y educación intercultural bilingüe.

» Los resultados fueron devueltos a las organizaciones, la información recogida permanece bajo su custodia.

\section{Resultados. Las voces de los agentes}

De acuerdo con la sugerencia de Coffey y Atkinson (1996), para pensar sobre el objetivo de una investigación es necesario decidir la codificación a partir de la cual se agruparán los datos para su posterior análisis. En este caso, se codificaron los datos del trabajo de campo en dos momentos de la investigación: en el 2017 y el 2019.

En el 2017, las entrevistas arrojaron tres códigos: cierre de escuelas, acceso a educación y educación intercultural bilingüe. En el 2019, se registraron tres códigos: cierre de escuelas, pedir apertura y educación intercultural bilingüe. Para la organización de las entrevistas se empleó el software Atlas ti. En la tabla 1 se presentan los resúmenes de los códigos.

Tabla 1.

Códigos 2017

\begin{tabular}{|c|c|c|c|}
\hline 2017 & $\begin{array}{c}\text { Cierre de las } \\
\text { escuelas }\end{array}$ & Acceso a la educación & $\begin{array}{c}\text { Educación intercultural } \\
\text { bilingüe }\end{array}$ \\
\hline Entrevista Ol & Malo & $\begin{array}{l}\text { Sí todos, pero dificulta } \\
\text { el traslado. }\end{array}$ & No hay docentes en kichwa \\
\hline Entrevista $\mathrm{O} 2$ & Pésimo & $\begin{array}{l}\text { Ahora queda lejos } \\
\text { para algunos. }\end{array}$ & El idioma se ha perdido. \\
\hline Entrevista O3 & Abuso del poder & Sí, pero todo está más lejos. & Todo se habla en español. \\
\hline Entrevista $\mathrm{O4}$ & $\begin{array}{l}\text { No pudimos } \\
\text { hacer nada. }\end{array}$ & $\begin{array}{l}\text { Todos acceden, algunos } \\
\text { les queda lejos. }\end{array}$ & $\begin{array}{l}\text { La enseñanza es en } \\
\text { el castellano. }\end{array}$ \\
\hline Entrevista 05 & Sin consulta & $\begin{array}{l}\text { Cuando el transporte no } \\
\text { cubre, eso trae problema. }\end{array}$ & Solo en el castellano. \\
\hline Entrevista $\mathrm{O6}$ & Sin consulta & Sí es completa & $\begin{array}{l}\text { Para todas las materias } \\
\text { no saben el idioma. }\end{array}$ \\
\hline Entrevista 07 & No queríamos & $\begin{array}{l}\text { Un problema, pero al } \\
\text { final todos asisten. }\end{array}$ & $\begin{array}{l}\text { Tratan de enseñar, } \\
\text { pero ni ellos saben. }\end{array}$ \\
\hline Entrevista O8 & Pésimo & Lejos, aunque todos van & Pocos docentes en kichwa. \\
\hline Entrevista O९ & No queríamos & $\begin{array}{l}\text { A los que les queda } \\
\text { lejos, lejos. Es dificil }\end{array}$ & $\begin{array}{l}\text { No hay libros para todas } \\
\text { las materias en kichwa. }\end{array}$ \\
\hline Entrevista 10 & $\begin{array}{l}\text { Incluso nos } \\
\text { resistimos }\end{array}$ & Sí tienen acceso & $\begin{array}{l}\text { Es más en castellano, } \\
\text { a la gente... }\end{array}$ \\
\hline Entrevista 11 & Malo & $\begin{array}{l}\text { Parece que pocos, } \\
\text { pero van todos. }\end{array}$ & $\begin{array}{l}\text { No hablan el kichwa } \\
\text { ya ni en la familia. }\end{array}$ \\
\hline Entrevista 12 & $\begin{array}{l}\text { No lo creímos, } \\
\text { pero pasó }\end{array}$ & Sí todos van & Sin material para el kichwa \\
\hline Entrevista 13 & Muy malo & Todos & No hay docentes \\
\hline
\end{tabular}

Fuente: elaboración propia con base en las entrevistas realizadas en el primer semestre del 2017. 
Al inicio de la investigación (2017), se halló que las medidas de segmentación territorial de los servicios educativos fue percibida como una arbitrariedad del Gobierno, ya que solo se comunicó a las comunidades, y no se les dio posibilidades de incidir en la resolución. Así, el Estado ejecutó la fusión de los servicios educativos y el cierre de algunos edificios donde funcionaban las escuelas.

Ya se escuchaba rumores, los del Ministerio de Educación se asomaban más veces por estos lados. Así, cerraron la escuela. Créame, que fue como un terremoto, cuando nos reunieron, pensábamos que nos harían caso, pero, no, todo lo que el gobierno quería lo hizo. (C1PI, 2017)

Cuando el Estado o las empresas, o quien sea, quieren poner un negocio, abrir una explotación minera, o agrícola; se consulta a las poblaciones indígenas, como dice la Constitución nueva. Pero, en algo tan, tan importante como la educación el cierre o apertura de las escuelas, no se nos consultó [...]. (Dirigente, 2019)

Nos opusimos, pero de nada sirvió... Incluso fuimos con oficios, acudimos hasta Quito... Sí, recibieron el oficio, ¡sí!... Y de qué sirvió, si sigue lo mismo. (C1Pa, 2016)

Se cerró, ahí están los escritorios, los bienes, la vieja bandera, da pena verla, ahí con los papeles alborotados, como si toda la historia anterior no valía, como si el esfuerzo hecho no significa nada... Porque debo decirle, cada ladrillo se levantó con dinero de la comunidad, con apoyo del Ministerio de Educación, sí también... Pero, con mingas de las familias, con sueños, con esperanzas. (C4PL, 2018)

El Estado otorgó un nuevo servicio escolar y las UE fueron implementadas entre los años 2013 y 2017. Fue una política pública creada para brindar enseñanza completa (más docentes por estudiantes), optimizar recursos y reorganizar la administración de servicios estatales educativos. Fue una medida "comunicada" a los poblados indígenas, pero - a decir de los entrevistados- no se discutió con ellos. En el Plan de Desarrollo de la Parroquia se registra la siguiente afirmación:

En educación, se cerraron 9 establecimientos educativos y se construyó la Unidad Educativa del Milenio Quizapincha, la dificultad se da por la falta de atención a los locales que se mantienen en funcionamiento y por la cantidad de estudiantes que han salido a estudiar fuera de la parroquia.

- En las 18 comunidades, disponen de un equipamiento básico, que por falta de mantenimiento se halla en un proceso de destrucción: casa comunal, canchas, iglesias, escuela, etc.

- Los 9 locales donde funcionaban las escuelas cerradas, se encuentran abandonadas y en malas condiciones. 
- Existe una organización y red social compleja de la comunidad, no se conocen los roles y funciones de las mismas, por lo que no se sabe de su intervención y beneficios. (GAD Kisapincha, 2015, p. 139)

Los vestigios del cierre de las escuelas permanecen y las construcciones donde funcionaban esas escuelas están abandonadas, aunque, según los informantes, existen gestiones para que sean entregados a las organizaciones comunitarias. En tal sentido, desde 2014 a la fecha de redacción del artículo, la situación de abandono de la infraestructura educativa anterior permanece; los entrevistados creen que estos edificios pueden servir para atender a personas vulnerables, particularmente a ancianos de la comunidad.

Una vez que se recopilaron y sistematizaron las entrevistas se evidenciaron tres aspectos. En primer lugar, el disgusto y la incomodidad producidos por el cierre de escuelas localizadas en el seno de las comunidades. En segundo lugar, aunque todas las personas entrevistadas manifestaron que el derecho al acceso a la educación se logró, las condiciones de distancia y los tiempos de traslado se vieron alteradas y causaron molestia. En tercer lugar, salieron a flote las dificultades de fortalecer la educación intercultural bilingüe, en particular la enseñanza del idioma indígena kichwa.

Sin embargo, los datos encontrados no señalaron nada nuevo. En el 2017 la Cepal había dado la alarma sobre el impacto negativo del cierre de escuelas rurales. Ya que nuestros datos coincidían, se decidió prolongar la indagación, en especial porque se acercaba un nuevo contexto marcado por el inicio de la presidencia de Lenin Moreno. Durante los meses que siguieron se comparó la información obtenida con otras investigaciones (Muyolema, 2018; Rodríguez Cahuana, 2016; Rodríguez Cruz, 2017); los hallazgos coincidían en la incomodidad de las poblaciones por el cierre de servicios educativos y la ausencia de diálogo con las comunidades indígenas y sus necesidades educativas.

Entre el 2018 y el 2019, se tensó la relación entre el Gobierno y los pueblos indígenas (Pinos Montenegro, 2019); en octubre del 2019 se produjo un nuevo levantamiento indígena y la Conaie reclamó, entre varios aspectos, la atención a las necesidades educativas de su población. Fue el momento propicio para volver a entrevistar a las personas, buscando determinar la recepción de la política pública de segmentación territorial educativa. Esta vez se partió de los datos ya obtenidos y surgieron los elementos que se muestran en la tabla 2.

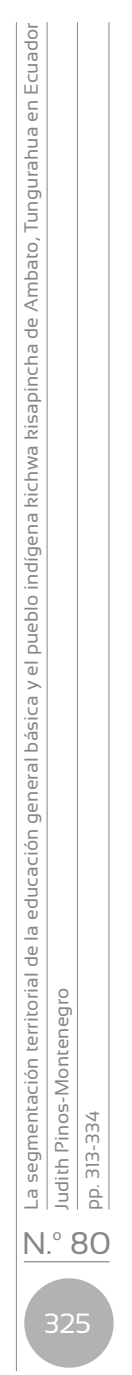


Tabla 2.

Códigos 2019

\begin{tabular}{|c|c|c|c|}
\hline 2019 & $\begin{array}{l}\text { Cierre de las } \\
\text { escuelas }\end{array}$ & Pedir reapertura & $\begin{array}{l}\text { Educación intercultural } \\
\text { bilingüe }\end{array}$ \\
\hline Entrevista Ol & Si fue malo & Ya no & $\begin{array}{l}\text { No hay docentes que } \\
\text { hablen kichwa. }\end{array}$ \\
\hline Entrevista $\mathrm{O} 2$ & Malo & Está de analizar & El idioma se ha perdido. \\
\hline Entrevista O3 & $\begin{array}{l}\text { En parte y parte, } \\
\text { bueno y malo }\end{array}$ & $\begin{array}{l}\text { Antes sí, ahora ya la } \\
\text { gente se organizó. }\end{array}$ & $\begin{array}{l}\text { Todo se habla } \\
\text { en español. }\end{array}$ \\
\hline Entrevista O4 & $\begin{array}{l}\text { Malo, pero no } \\
\text { pudimos decidir }\end{array}$ & No & $\begin{array}{l}\text { La enseñanza es } \\
\text { en el castellano. }\end{array}$ \\
\hline Entrevista O5 & Malo & No & Solo en el castellano. \\
\hline Entrevista O6 & Sin consulta & Ya no tiene sentido. & $\begin{array}{l}\text { Para todas las materias } \\
\text { no saben el idioma. }\end{array}$ \\
\hline Entrevista O7 & $\begin{array}{l}\text { Hicimos petición } \\
\text { de reapertura } \\
\text { al Ministerio }\end{array}$ & Ya se acomodó todo. & $\begin{array}{l}\text { Tratan de enseñar, } \\
\text { pero ni ellos saben. }\end{array}$ \\
\hline Entrevista ○८ & $\begin{array}{l}\text { Malo y también } \\
\text { bueno; porque } \\
\text { ahora tienen nuevos } \\
\text { profesores }\end{array}$ & $\begin{array}{l}\text { Ya nos reunimos con } \\
\text { el Ministerio, pero } \\
\text { hay que volver a } \\
\text { consultar a todos. }\end{array}$ & $\begin{array}{l}\text { Pocos docentes } \\
\text { en kichwa }\end{array}$ \\
\hline Entrevista O९ & No queríamos & $\begin{array}{l}\text { Ya no son los mismos } \\
\text { grupos de familias }\end{array}$ & $\begin{array}{l}\text { No hay libros para todas } \\
\text { las materias en kichwa. }\end{array}$ \\
\hline Entrevista 10 & $\begin{array}{l}\text { Incluso nos } \\
\text { resistimos }\end{array}$ & No, hay que consultar & $\begin{array}{l}\text { Es más en castellano, } \\
\text { a la gente... }\end{array}$ \\
\hline Entrevista 11 & Malo & No & $\begin{array}{l}\text { No hablan el kichwa } \\
\text { ya ni en la familia }\end{array}$ \\
\hline Entrevista 12 & $\begin{array}{l}\text { No lo creímos, } \\
\text { pero pasó }\end{array}$ & $\begin{array}{l}\text { Estamos pensando } \\
\text { en consultar }\end{array}$ & $\begin{array}{l}\text { Sin material para } \\
\text { el kichwa }\end{array}$ \\
\hline Entrevista 13 & - & - & $\begin{array}{l}\text { - (no fue posible } \\
\text { entrevistarlo) }\end{array}$ \\
\hline
\end{tabular}

Fuente: elaboración propia, entrevistas realizadas en el último trimestre del 2019.

Un aprendizaje del trabajo de campo fue que la perspectiva de los agentes puede cambiar en corto tiempo, aunque existan también continuidades. Tras la segunda indagación (2019), se evidenció una resignación y aceptación de la política pública de segmentación territorial de los servicios educativos y se detectaron la incomodidad con el acceso cultural y la ruptura de vínculos comunitarios más particulares.

La educación ecuatoriana tiene en su base legal el mandato de garantizar el acceso a los servicios educativos y en el artículo 6 de la LOEl, se señala: 
Art. 6. Obligaciones. La principal obligación del Estado es el cumplimiento pleno, permanente y progresivo de los derechos y garantías constitucionales en materia educativa, y de los principios y fines establecidos en esta Ley. El Estado tiene las siguientes obligaciones adicionales:

a. Garantizar, bajo los principios de equidad, igualdad, no discriminación y libertad, que todas las personas tengan acceso a la educación pública de calidad y cercanía [...]

Sin embargo, en el caso que estudiamos, la medida estatal de segmentación territorial educativa rompió el principio de "cercanía", al cerrar escuelas y concentrar los nuevos servicios lejos de algunas comunidades, donde la mayor parte del personal docente no habla el idioma kichwa. En consecuencia, los niños de las comunidades se trasladan, para recibir educación, a lugares ajenos (física y culturalmente) a sus comunidades. El cierre de las escuelas se leyó como un hecho contrario al espíritu de la ley y causa incomodidades en las comunidades indígenas.

En la escuela hacíamos reuniones. Cuando iban, cuando volvían, para acordar las mingas, todo pasaba por la escuela... Ahora dijeron que era mejor, que tendrían más profesores, carro y comida. ¡Vea, ahora esta desgracia! Ya libros no hay, la comida son galletas y galletas que se cansan los wawas de comer lo mismo, el transporte bueno, van como sardinitas, cuando pueden, sino a caminar lejos... De nuestras ideas, nada... ¿Cuándo han hecho valer nuestros pensamientos? ¡Nunca!... (C3PS, 2017)

La escuela casi al frente de mi casa, ¿qué caminaban? Nada... Yo estaba pendiente, cuando regresaba del mercado, cuando pasaba con mis cosas... Sabía dónde estaban. No quisimos que cierren... Educar acá, con nuestras costumbres, acá cerca de todo... (C2PL, 2017)

La compañera le puede contar... Su propia hija tiene que caminar, hasta donde pasa el bus de la Unidad Educativa, pero un día no llegó, ni a la unidad educativa, ni a la casa. ¿Qué había pasado? Tienen que caminar como media hora, hasta esperar el bus escolar. Pero, el bus pasó antes, así que los wawitos se perdieron...

¿Cómo se perdieron?

Empezaron a caminar rumbo a la escuela, pero eso es lejos, no llegaron. Luego, regresaron a casa, pero no llegaron. Se reunió la comunidad y los encontraron, sin comer, Ilorando, perdidos. (C7PL, 2017)

A decir de los entrevistados, la cercanía al centro educativo proporcionaba tranquilidad, optimizaba el tiempo de traslado, fortalecía las relaciones entre personas conocidas. La escuela era —es— más que un sitio donde los niños recibían educación. En el caso de las once comunidades, en la escuela se tejían lazos sociales fuertes, era el sitio de encuentro de las 
familias y de acuerdos comunitarios. Un espacio donde todos se conocían y donde la organización Ilamada Cabildo observaba el cumplimiento de la jornada laboral por parte de los docentes. El cierre de la escuela puede interpretarse como una desposesión de un derecho y una afectación a las comunidades y sus habitantes. En estos años, el contexto parroquial cambió y los servicios estatales — como ya se dijo, originalmente eran quincese consolidaron en cuatro UE (figura 1). Hay poco personal docente que domina las lenguas indígenas, pero al parecer es un problema no solo ecuatoriano, sino global como lo ha documentado la Unesco (2019), aspecto que profundiza la desaparición de las lenguas nativas.

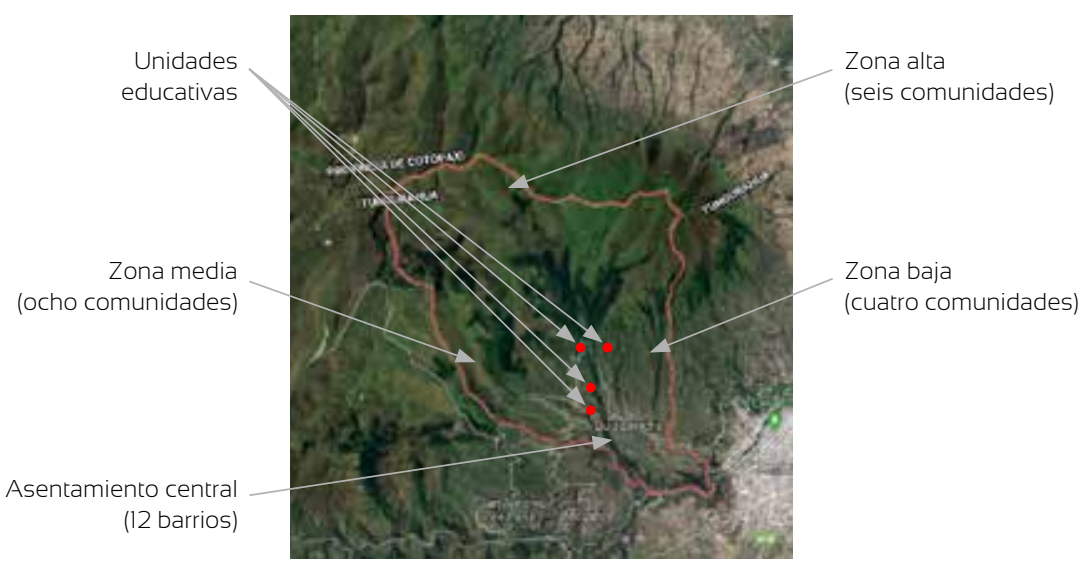

Figura 1. Parroquia Kisapincha (Quizapincha)

Fuente: Google Maps. https://n9.cl/jOsx

En la figura 1 se observan los 119,3 km² del territorio de Kisapincha, los puntos rojos son los cuatro servicios educativos actuales (UE) y las flechas señalan las áreas geográficas en las que están divididos según el Gobierno Autónomo Parroquial de Kisapincha (zona alta, media y baja); donde antes existían escuelas, ahora todas se concentran en la parte media y baja.

Lo primero era el número de niños en cada aula. Pasaron a ser demasiados, eran como 50 en un caso. Mi hijo se sentía agobiado por el espacio cerrado, por tanta gente junta. Pero, hace casi dos años, parece que todo mejoró, el sistema de cupos, distribuye a los estudiantes casi como "equilibrado", ahora en cada escuela. Ahí pasa, que están adecuados. Pero, también hay quienes no están cerca de su casa. (HTK 1, 2019)

Bueno, ya estamos como organizados, le diré... Los carros recorren las comunidades y llevan a los wawas a clases. Cuando algo falla, tenemos lo que Ilamamos un taxi amigo. El problema es cuando no hay quien pague el taxi [ríe]. 
¿Pasa?

Bueno, hay gente pobre, pobre... Pero, de alguna manera se arregla la cosa (EN13, 2018)

En el último levantamiento, la Conaie pidió la reapertura de las escuelas, como sabe; hubo muertos, el gobierno se asustó; luego, a la otra semana los del Ministerio de Educación vinieron, se reunieron con nosotros; pero, ya reunidos todos, nadie dijo re-abran las escuelas... Acordaron volver y que iban a ver, a analizar, dicen... Vea, ya, ya estamos. Otra vez, deshacer es grave. (EN 11, 2019)

Como ya se mencionó, en octubre del 2019 los pueblos indígenas protestaron contra el actual Gobierno. La Conaie pidió, entre otros aspectos, la reapertura de las escuelas (Conaie, 2018, 2019). En ese contexto, resultó oportuno preguntarse, si los reclamos e incomodidades detectadas en el año 2017, seguían vigentes. El trabajo de campo de esos meses arrojó que no, porque las rutinas de vida (horarios, transporte, alimentación y otras) ligadas a la cotidianeidad escolar se habían regulado y la ampliación de la obligatoriedad de la educación general básica, es bien recibida. No obstante, hay un aspecto de incomodidad que se mantiene. La estructura educativa establecida por el Estado en el formato de UE, responde a una forma nacional homogénea y a un enfoque curricular único. Sin embargo, la diversidad étnico-cultural del Ecuador requiere abarcar también las necesidades educativas de pueblos y nacionalidades indígenas, que tienen, entre otros, el derecho de educarse en lengua propia; para eso se crearon las uE bilingües (como se las llamó) que deben brindar la educación en lenguas indígenas y fortalecer sus saberes.

Hasta la fecha, los libros que reciben son en español. El kichwa, quedó para el saludo, la propaganda, en el día a día, nada... Todo en español, en eso, nada, no hay progreso... (Dirigente indígena, 2018).

Si bien la Educación Intercultural Bilingüe necesita apoyo... El Estado, no lo hizo. Le pongo un ejemplo, ¿qué cantidad de docentes hay que dominen ambos idiomas (español y kichwa)?, muy pocos. Como hay que cubrir los puestos, son los mestizos, que no saben nada de kichwa, los que ocupan los cargos de profesores. Entonces, junto al cambio, no se fortaleció la Eı a nivel superior, no se produjo — pronto libros en idiomas ancestrales, no se reconstruyó ni discutió un currículo propio. ¿Qué hizo el gobierno? Impuso su visión, un currículo que dice debe ser nacional e internacional, con eso de la PISA, de lo Ineval... (Dirigente indígena, 2018)

En este aspecto, hay malestar respecto a la ausencia de recursos didácticos, personal docente y otros que fortalezcan la enseñanza de la lengua nativa. Mientras que la mayoría de libros de texto se hayan desarrollados para todos los niveles en español, no sucede lo mismo con el kichwa. 


\section{Conclusiones}

Si bien en el Ecuador el 6,7\% de los habitantes se autoidentifica como indígena, en la parroquia Kisapincha ese porcentaje es del 70,86 \%, por lo cual resulta un territorio ideal para recuperar la perspectiva de los agentes indígenas respecto a un hecho social. Esta investigación se propuso identificar cómo recibió el pueblo kichwa kisapincha el sistema de segmentación territorial educativa, de los servicios estatales. Esta pregunta central se derivó en dos interrogantes: 1) ¿Qué cambios perciben los pueblos indígenas respecto al acceso a la educación tras el proceso de segmentación territorial? 2) ¿Qué aspectos en torno a la segmentación educativa reclaman los pueblos indígenas?

Respecto a la pregunta central, podemos evidenciar dos momentos. En el primero, que corresponde al año 2017, la segmentación territorial educativa se recibió como un proceso arbitrario que no consideró las perspectivas de las organizaciones indígenas. En el segundo, en 2019, si bien la molestia subsiste, ahora se interpreta como un proceso que difícilmente tendría reversibilidad, tanto porque ya están familiarizados con los mecanismos de acceso físico a las UE, como porque el sistema de asignación de cupos escolares para instituciones públicas ha mejorado. En resumen, el acceso físico está garantizado, no así el acceso con pertinencia cultural.

En cuanto a los cambios que perciben las personas entrevistadas, pueden esquematizarse en que las UE son calificadas como servicios más completos, pero que aglutinan a más individuos diversos, lo cual trae como consecuencia que el vínculo comunitario se haya debilitado. Por ejemplo, en una de las UE ahora confluyen estudiantes de seis comunidades de la zona alta, por lo que se han roto los viejos circuitos de comunicación escuela-comunidad-familias, que antes de la actual segmentación territorial sobrepasaba el hecho educativo. Poco a poco la gente se resigna y se acomoda a la actual situación, hoy la escuela ya no es el sitio de reunión comunitaria. El cuerpo docente de las escuelas cerradas no perdió su trabajo, sino que, al igual que en todo el territorio nacional, fue reubicado según las necesidades del Estado y la perspectiva de optimización de recursos (Pinos Montenegro, 2017).

En cuanto a los reclamos, existen varios cuestionamientos. El primero tiene relación con el acceso cultural, es decir, se cuestiona que el hecho educativo cotidiano se desarrolle solo en español y se demanda también la existencia de materiales en lenguas indígenas y de docentes que dominen ambos idiomas. ¿Por qué faltan docentes que dominen lenguas indígenas? Al ejecutar los cambios legales (LOEl, 2011, Acuerdo Ministerial 353-13) y eliminar escuelas multigrados y unidocentes (generalmente localizadas en zonas rurales lejanas), en las UE requieren más personal docente en 
diferentes áreas del conocimiento, plazas laborales que son cubiertas por profesionales (incluso sin formación pedagógica), a través del concurso público Ser Maestro, de tal manera que ampliar la escolaridad trajo también la necesidad de más docentes, y solo ahí se evidenció el déficit en la formación de profesionales con dominio de idiomas nativos. Hasta ahora no hay un estudio específico de cuántos docentes bilingües se requieren en Ecuador, esperamos realizar tal indagación durante los siguientes años.

En tal sentido, estas demandas tienen continuidad histórica y, al parecer, el Estado ecuatoriano sigue en deuda con los pueblos indígenas en cuanto a la necesidad de más docentes que dominen idiomas indígenas. Se sugiere una relación con lo que la Unesco documentó ampliamente en el estudio realizado por Mato (2015): no hay programas de educación superior suficientes (en número y cobertura geográfica) para la formación de docentes en lenguas indígenas. El segundo cuestionamiento tiene relación con el abandono de la infraestructura de las escuelas que permanecen cerradas y que pueden ser utilizadas por las comunidades para otros propósitos, como las reuniones comunitarias, así lo ratifica el Gobierno parroquial de Kisapincha. Tenemos la hipótesis de que tal situación se repite en todo el país y requeriría un análisis específico del estado actual de la infraestructura que dejó de emplearse en el funcionamiento de escuela unidocentes y multigrado.

Así, esta investigación permite entender que el acceso físico a una educación no es suficiente. Las poblaciones reflexionan sobre el tipo de contenidos, su pertinencia cultural, también sobre las posibilidades y limitantes que crea un proceso de segmentación territorial. La única forma de escapar de los designios estatales, en el caso ecuatoriano, es poseer suficientes recursos para costearse una educación privada. De tal manera que el derecho al acceso a la educación queda relativizado por la oferta estatal, la cual tiene serias limitaciones en el caso de las demandas bilingües (lengua nativa y español). La oferta educativa se dice intercultural y bilingüe, pero en ella sigue primando el idioma español, los materiales en español y faltan docentes que dominen kichwa-español; son esos los desafíos y deudas que el Estado central debe asumir.

Esta investigación también abre nuevas preguntas: ¿Qué percepciones tienen las otras comunidades indígenas de la misma provincia? ¿Cómo un currículo nacional único se aplica en instituciones privadas? ¿Por qué el estado tiene dificultad en la contratación de docentes que dominen idiomas nativos? ¿Cuántas ofertas académicas para formación de docentes en idiomas nativos hay en Ecuador? ¿Cómo funciona la educación intercultural bilingüe en el actual contexto? ¿Qué pasó con la infraestructura de las instituciones cerradas? Son aspectos que quedarán para las siguientes investigaciones. 


\section{Referencias}

Agrupación de hermanos en planteles fiscales depende de disponibilidad de cupos, dice Ministerio de Educación. (2018, 29 de agosto). El Comercio. https://www.elcomercio.com/actualidad/agrupacion-hermanos-plantelesfiscales-disponibilidad-cupos.html

Archer, M. (2009). Teoría social realista. El enfoque morfogenético. Ediciones Universidad Alberto Hurtado.

Asamblea Constituyente. (2008). Constitución política del Ecuador.

Asamblea Nacional. (2011, 31 de marzo). Ley Orgánica de Educación Intercultural. Registro Oficial, 417.

Bourdieu, P. y Passeron, J. C. (2013 [1970]). La reproducción. Elementos para una teoría del sistema de enseñanza. Siglo xxı.

Confederación de Nacionalidades Indígenas del Ecuador-Conaie. (2018, 28 de septiembre). Categoría: nacionalidades-sierra. https://conaie. org/category/nacionalidades-sierra/

Confederación de Nacionalidades Indígenas del Ecuador-Conaie. (2018, 22 de febrero). Conaie realizará asamblea plurinacional por la restitución de la educación. https://conaie.org/2018/02/22/conaie-realizara-asamblea-plurinacional-la-restitucion-la-educacion-bilingue/

Coffey, A. y Atkinson, P. (1996). Making sense of qualitative data: Complementary research strategies. Sage Publications.

Di Caudo, M. V., Llanos Erazo, D. y Ospina, M. C. (2016). Interculturalidad y educación desde el Sur. Contextos, experiencias y voces. Abya Yala/ Universidad Politécnica Salesiana, Clacso, Cinde, Universidad de Mazinales.

Dubet, F. (2011). Repensar la justicia social. Siglo xxı.

Fuentes Reverón, S. (2017). El acceso a los servicios: consideraciones teóricas generales y reflexiones para Cuba. Revista Cubana de la Salud Pública, 272-286.

GAD Kisapincha. (2015). Plan de Desarrollo y Ordenamiento Territorial del Gobierno Autónomo Parroquial de Kisapincha. Ambato.

Haboud, M. (1998). Quichua y castellano en los Andes ecuatorianos. Abya Ayala.

Hernández Samperi, R., Fernández Collado, C. y Baptita Lucio, P. (2012). Metodología de la Investigación. McGraw-Hill.

Instituto Nacional de Evaluación Educativa-Ineval. (2016). Resultados educativos, retos hacia la excelencia.

Instituto Nacional de Evaluación Educativa-Ineval. (2018). La educación en el Ecuador. Logros alcanzados y nuevos desafíos. Resultados educativos 2017-2018. Isch, E. (2011). Las actuales propuestas y los desafíos en Educación. Educação \& Sociedade, 32(115), 373-391. 
Krüger, N. (2012). La segmentación educativa argentina: reflexiones desde una perspectiva micro y macro social. Páginas de Educación, 137149. https://doi.org/10.22235/pe.v5i1.605

León, M. (2017). Protección social de la niñez en el Ecuador. Cepal-Unicef. https://repositorio.cepal.org/bitstream/handle/11362/41233/ S1700082_es.pdf?sequence=1\&isAllowed=y

Luna Tamayo, M. (2017). Políticas públicas en educación y participación ciudadana. La experiencia del contrato social por la educación. Polemika, 1(2), 50-55. https://revistas.usfq.edu.ec/index.php/polemika/ article/view/324

Massey, D. (2008). Geometrías internacionales del poder y la política de una ciudad global: pensamientos desde Londres, conferencia en Cendes. Tercera Época. Universidad Central de Venezuela.

Mato, D. (2015). Pueblos indígenas, Estados y educación. Aprendizajes en varios países de América Latina potencialmente útiles a los procesos en marcha en Argentina. Cuadernos de Antropología Social, 41, 5-23.

Ministerio de Educación. (2018). Optimización de la planta docente busca mejorar la calidad en educación. https://educacion.gob.ec/optimizacion-de-la-planta-docente-busca-mejorar-la-calidad-en-educacion/

Muyolema,A.(2018). LacreacióndelaSecretaríadeEducaciónIntercultural, ni restauración, ni autonomía. Línea de fuego. https://lalineadefuego. info/2018/08/14/la-creacion-de-la-secretaria-de-educacion-intercultural-ni-restitucion-ni-autonomia-por-armando-muyolema/?fbclid=IwAR04_3JQ0gpwQ9ku7_UWotd6fR4c3a-noEZL8bEJMo0CoSvRMkh7m1utZms.

Organización de las Naciones Unidas para la Educación, la Ciencia y la Cultura-Unesco. (2019). Atlas de las lenguas del mundo en peligro. http://www.unesco.org/new/es/communication-and-information/access-to-knowledge/linguistic-diversity-and-multilingualism-on-internet/atlas-of-languages-in-danger/

Pinos Montenegro, J. (2017). La sindicalización docente. Entre el derecho a la huelga y la prohibición de limitar un servicio público. Contribuciones a las Ciencias Sociales, 25-45.

Pinos Montenegro, J. (2019). Ecuador se levanta. La lucha indígena continúa, a pesar de la represión estatal. http://www.iri.edu.ar/index. php/2019/10/11/ecuador-se-levanta-la-lucha-indigena-continua-a-pesar-de-la-represion-estatal/.

Ponce Jarrín, J. J. y Acosta, A. (2010). La pobreza en la "revolución ciudadana" o ¿pobreza de revolución? Debate-Flacso Andes, 7-20.

Problemas con asignación de cupos en escuelas persisten. (2019, 5 de agosto). La Hora. https://www.lahora.com.ec/tungurahua/noticia/1102263297/ problemas-con-asignacion-de-cupos-en-escuelas-persisten 
Ringer, F. (1990). Dos culturas académicas: Francia y Alemania en torno a 1900. http://www.educacionyfp.gob.es/dam/jcr:cdbb1847-e8644cbd-9885-aa4ac3ac1812/re199005-pdf.pdf

Rodríguez Caguana, A. (2016). Los derechos lingüísticos de los pueblos indígenas del Ecuador ¿interculturalidad o asimilación? (2007-2014). Debates-Flacso Ecuador, 113-124.

Rodríguez Cruz, M. (2017). Construir la interculturalidad. Políticas educativas, diversidad cultural y desigualdad en Ecuador. Íconos, Flacso Ecuador, 50-78.

Secretaría Nacional de Planificación del Desarrollo-Senplades. (2017). Plan Nacional de Desarrollo 2017-2020. Toda una vida. Stake, R. E. (2005). Investigación con estudio de casos. Morata.

Vasilachis de Gialdino, I. (2013). Estrategias de la investigación cualitativa. Gedisa Editorial.

Verhoeven, M. (2013). Desigualdades múltiples, carreras escolares y pruebas en sistemas educativos post-masificación. Propuesta Educativa, 22(40), 87-98.

Weber, M. (1964). The Theory of social and economic organization. Free Press. 